\title{
BIRTH TRAUMA TO THE VERTEBRAL ARTERIES
}

\author{
BY \\ P. O. YATES \\ From the Department of Pathology, University of Manchester
}

(RECEIVED FOR PUBLICATION APRIL 11, 1959)

Birth is a very traumatic event. This is particularly emphasized by the frequency of intracranial haemorrhage and cerebral damage in the newborn infant. However, the greatest manipulative strain in all methods of delivery is imposed on the infant's neck and it is therefore surprising that there have been very few investigations of this region except following breech delivery. Moreover none of the reports records the effects of trauma on the cervical portions of the vertebral arteries, two of the principal vessels supplying the brain. It is the purpose of this paper to draw attention to traumatic lesions in the necks of infants delivered by a variety of methods who died round about the time of birth, and to indicate their possible significance in the production of damage to the brain.

\section{Material and Methods}

The infants examined were selected during one year from 213 stillborn and neonatal deaths occurring in 2,800 births. The only cases excluded were malformed foetuses, such as hydrocephalics or anencephalics, and those who, judging by the degree of maceration, had been dead in utero before labour started. With these exceptions the necks were removed from 60 foetuses chosen at random and were examined without any knowledge of the findings in the rest of the autopsy or of the method of delivery.
The 60 cases included eight breech deliveries and three by caesarean section. Twenty were more than two weeks premature and eight had Rhesus antibody disease; 16 were stillborn.

The cervical vertebrae were removed intact, usually from $\mathrm{C} 7$ to the foramen magnum. Occasionally lower vertebrae were included and the foramen magnum itself. The specimen was fixed in $10 \%$ formol saline after which it was placed in $6 \%$ nitric acid for 24-48 hours to allow decalcification. It was divided in horizontal planes into five to seven blocks, which were then embedded in paraffin wax. Histological sections stained by a variety of methods were examined from different levels. Occasionally serial sections were examined.

Since it was not possible to examine every joint, ligament or nerve root histologically, the lesions described below must be considered the minimum number present.

\section{Results}

Evidence of distortional trauma to the cervical spine was found in 27 cases. The lesions were of three main types: extradural and subdural haemorrhages which lay commonly beneath the ligaments of the arch and were often very extensive (Fig. 1); haemorrhages into joint capsules and synovial villi (Fig. 2); torn ligaments and dura (Fig. 3). These changes were present following all methods of delivery including caesarean section. They were in general most severe after breech delivery.

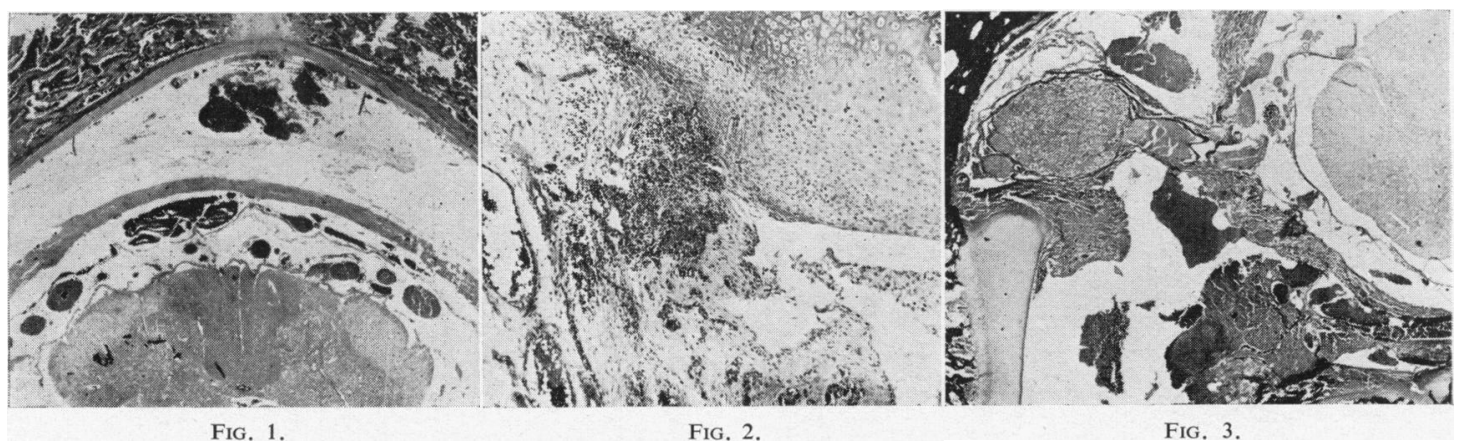

Fig. 1.-Transverse section of cervical spine showing subdural haemorrhage and haemorrhage into the tissues beneath the vertebral arch. FIG. 2.-Joint capsule showing haemorrhage and some tearing. $\times 20$. FIG. 3.-A disrupted ligament lies beneath the posterior nerve root. 


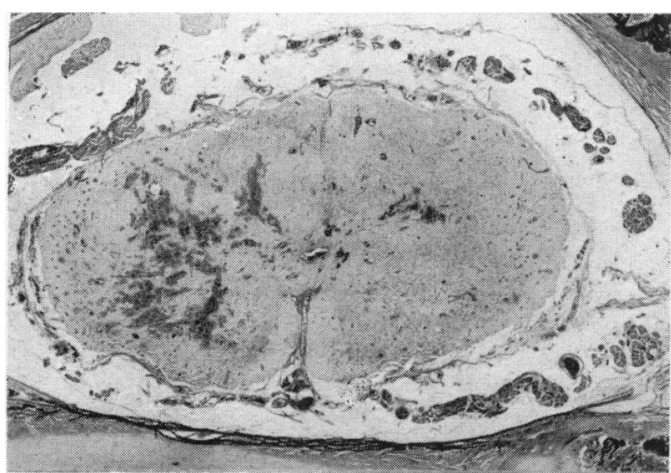

Fig. 4.-Spinal cord showing contusion.

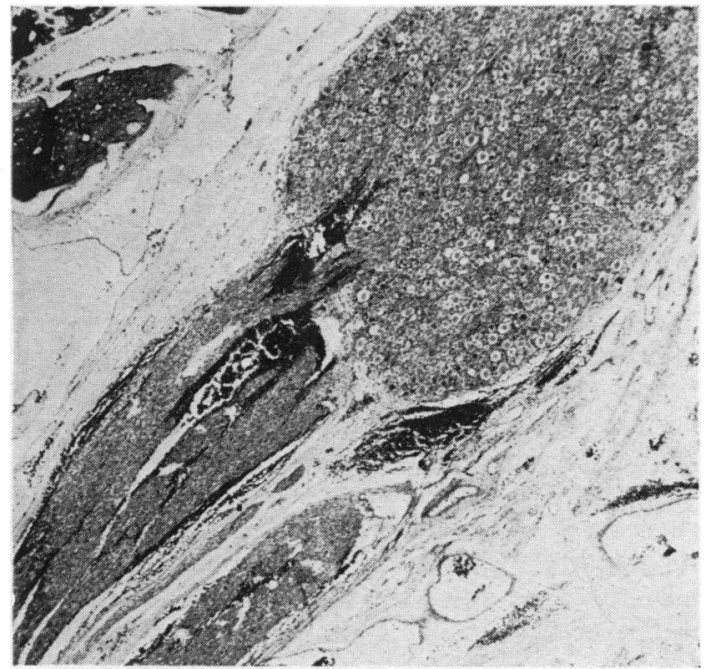

FIG. 5. -The dorsal nerve root has been partially torn as it leaves the posterior root ganglion. $\times 30$.

Direct damage to the spinal cord was not common. In only two cases, both breech deliveries, was there extensive bruising and destruction of the cord

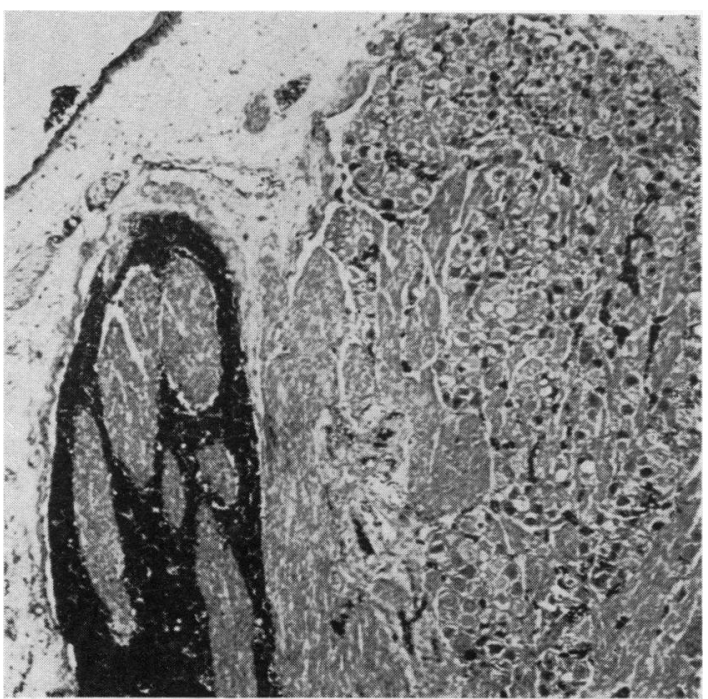

FIG. 6.-Extensive haemorrhage into torn anterior nerve root. $\times 50$.

(Fig. 4). Occasional haemorrhages of no obvious significance per se were found in other cases. On the other hand, bruising and tearing of spinal nerve roots was found in nine cases, the anterior root being slightly more frequently affected than the posterior. In general the nerve roots of one side only were affected (Figs. 5 and 6).

Since the vertebral arteries are of vital importance to the brain, evidence was sought of damage or obstruction to these vessels in their course through the transverse processes of the cervical vertebrae. In 24 cases there were haemorrhages in the adventitial coat of one or both arteries. These varied in size, some small and crescentic, others large and either surrounding the vessel or reducing its lumen by compression from one side (Figs. 7, 8 and 9). They extended for up to $1 \mathrm{~cm}$. along the wall of the vessel. The vertebral artery in its canal is accom-

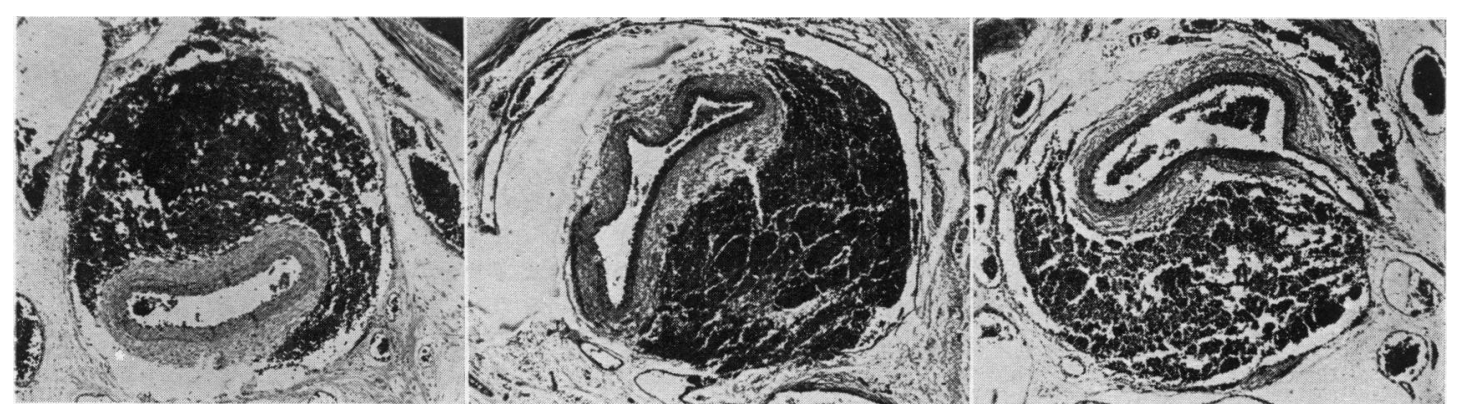

FIG. 7.

FIG. 8 .

FIG. 9.

FIG. 7-Haematoma almost surrounding a vertebral artery $\times 20$.

FIG. 8.-Compression of a vertebral artery by a large crescentic haematoma. $\times 20$.

Fig. 9.- Haematoma extending from the neighbourhood of the origin of a branch from a vertebral artery. $\times 20$. 
panied by many large thin-walled venous sinuses. These also were ruptured in some cases with haemorrhage into loose connective tissue of the transverse foramen. However, the haematomas in the wall of the artery were usually enclosed within a collagenous outer adventitial layer. This outer adventitia is important in preventing over-distension of the vessel and it may therefore be expected to resist any outward expansion required to accommodate a haematoma. Serial sections of some of these mural haematomas indicated that they arose from tearing of the branches of the vertebral artery at their junction with the major trunk (Figs. 9 and 10). Nowhere in the sections examined was the main vessel itself torn.

In one baby, who died 12 days after birth, the left vertebral artery was found to be completely occluded by thrombus as it passed around the first cervical vertebra (Fig. 11). This thrombus showed evidence of early organization and appeared to be about three to four days old. It is not possible therefore to attribute the thrombosis to birth injury with any certainty, though other evidence of trauma was present in the neck.

\section{Discussion}

It is not the intention in this paper to correlate the findings closely with a detailed analysis of the techniques of delivery or with the pathological changes in the rest of the autopsy, but to emphasize the frequency of the lesions described in a random group of perinatal deaths.

Direct Trauma to Spinal Cord. The occurrence of birth injury to the brain and its clinical effects

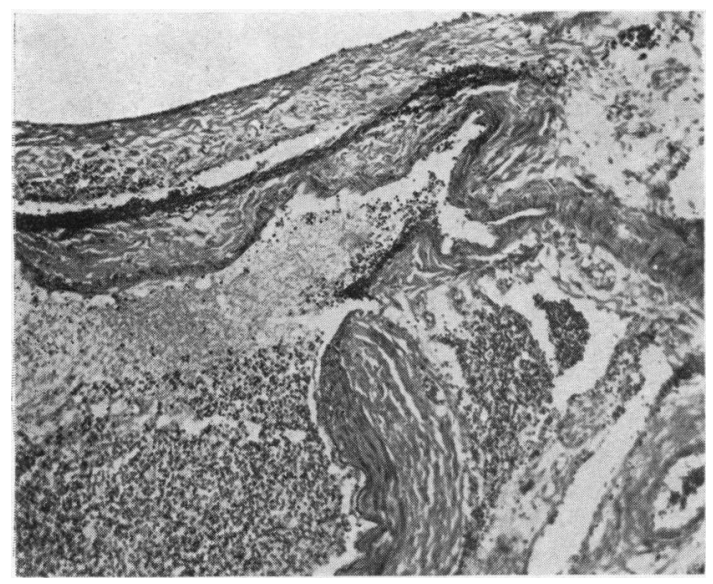

FIG. 10.-A tear in the wall of a branch of a vertebral artery at its origin from the parent vessel. Blood has escaped into the angle between the vessels and is continuous with a much larger haematoma in this section. $\times 70$.

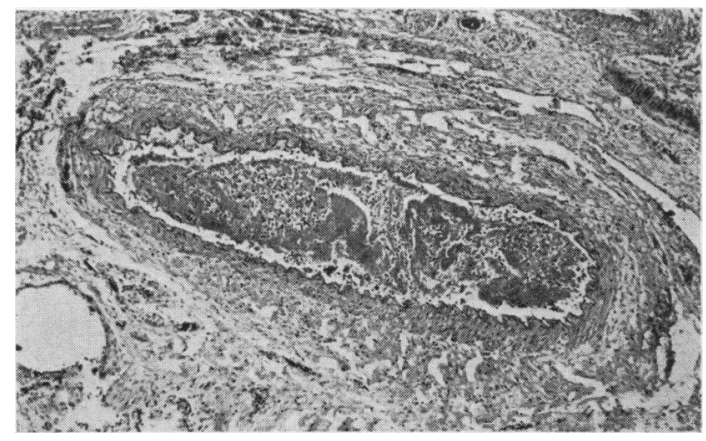

FIG. 11.-Vertebral artery occluded by thrombus. $\times 30$.

were well appreciated a century ago by Radford (1834) and Little (1843). Little (1862) was aware also of damage more particularly to the brain stem and spinal cord and stated that difficulty in deglutition and respiration resulted from damage to the medulla. Injury to the neck is especially liable to occur during breech delivery, and Pierson (1923) recorded 17 cases of fracture or dislocation of vertebrae in 36 deaths. Disruption of the spinal cord itself was reported by Ford (1925) in six cases of breech delivery. In contrast Crothers and Putnam (1927), reviewing birth injuries to the spinal cord, concluded that serious cord damage was rare after vertex presentation. However, minor haemorrhages into the substance of the cord have been found after all methods of delivery. Hausbrandt and Meier (1935) examined the spinal cord in 103 premature and full-time foetuses for whom there was either no obvious cause of death or a clinical diagnosis of intra-uterine asphyxia or inhalation of liquor had been made. They found petechiai haemorrhages in $30 \%$ of the spinal cords, especially in the upper cervical region. Hirvensalo (1949) found that small haemorrhages were present in medulla and pons in $37 \%$ of all premature and $50 \%$ of full-term foetuses.

In the present series a significant degree of traumatic bruising of the cervical cord was found in only two cases, both breech deliveries (Fig. 4). The mode of origin of smaller petechial haemorrhages is not clear. Whilst they may be a manifestation of general anoxic damage to capillaries, Baar (1946) has pointed out that such 'asphyxial' haemorrhages are often indistinguishable from those produced by the great intra- and extra-vascular pressure differences in the presenting parts during labour, a factor which would account for their frequent occurrence after vertex presentation.

Damage to Spinal Nerve Roots. In nine cases tearing and bruising of spinal nerve roots was found 
and occasional arterial twigs from the vertebral arteries supplying the nerve roots were found to be torn. Such lesions may of course result in a fully developed Erb or Klumpke type of paralysis. Where the third and fourth cervical roots are disrupted then a complete phrenic palsy may be obvious; simple bruising or ischaemic damage would result in temporary respiratory difficulty through dysfunction of the diaphragm.

It is widely held that congenital torticollis is the result of tearing of the muscle belly of the sternomastoid during delivery. This was evidently not the opinion of Little (1862) whose Cases 48 and 49 had wrynecks. He thought the condition was due to spasm of the muscle following injury to the neck. Chandler (1948) treated 225 cases surgically, 26 of whom were under 4 months of age. In the excised specimen he found degenerating muscle and fibrosis but no evidence of an old haematoma. Roemer (1954) followed up 44 cases and found four with persistent associated palsies. One had an Erb's palsy, one a facial paralysis and two had scoliosis. It is possible in many cases that the underlying lesion in congenital torticollis is partial denervation of the sternomastoid muscle with spasm and subsequent muscular atrophy and contracture.

Damage to the Vertebral Arteries. The intramural haematomas found in the vertebral arteries of 24 foetuses may be a very important cause of perinatal morbidity and mortality. Where the arterial obstruction is of moderate degree the infant may survive to show later evidence of ischaemic cerebral damage.

It is clear that in normal circumstances intramural haemorrhages, since they cannot be formed at more than systolic blood pressure, only compress the lumen significantly if a valve pocket is produced by dissection of the wall from the lumen. However, the situation during delivery of the head and neck of an infant is quite different. The presenting parts may be at or near atmospheric pressure whilst the rest of the body is subject to the additional pressure of the contracting uterus, perhaps $200 \mathrm{~mm}$. $\mathrm{Hg}$. The blood pressure is therefore raised from the normal $90 / 50$ to $290 / 250 \mathrm{~mm}$. $\mathrm{Hg}$, the pulse pressure remaining the same. With the tissues of the neck and head at normal atmospheric pressure and the intravascular pressures very considerably raised any slight tear of vein or artery will produce a haematoma. If the haemorrhage has occurred within the tough adventitia of the vertebral artery the haematoma will be unable to disperse rapidly. On completion of delivery the intravascular arterial pressure returns to normal levels, which may be inadequate to maintain the lumen of the artery past a tense intramural haematoma formed at a pressure of $290 \mathrm{~mm}$. Hg. By such a mechanism vertebral bloodflow may be seriously embarrassed for a period of minutes or hours, which is sufficient for permanent cerebral ischaemic damage to be caused. Furthermore, disruption of the adventitial coat may affect the autonomic innervation of the vertebral artery producing for a time spasmodic and irregular contractions.

The vertebral arteries are unique in that they unite to form a common basilar arterial trunk which then gives off bilaterally symmetrical branches. Because of this arrangement obstruction of one vertebral artery may be sufficient to produce bilateral lesions of the brain stem, cerebellum and occipito-temporal regions (Hutchinson and Yates, 1957).

Obstruction to the vertebral arterial flow is particularly significant at the time of birth since it is at this period that the vegetative functions of the brain stem are established. This is emphasized by the observation of Himwich and Fazekas (1941) that in neonatal dogs the metabolic activity, as measured by oxygen demand in vitro, was much lower in the cerebral hemisphere than in the cerebellum or medulla. In later life the reverse was true. Similar observations were made on tissue from human infants by Racker (1942).

The effects of ischaemia of the brain stem may be evident as cardio-respiratory failure; in the words of Radford (1834) 'the difficulty of commencing respiration does not depend upon a mechanical obstruction, from mucus in the trachea; but on the injury which the brain has suffered'. This may be associated with difficulty in deglutition (Little, 1862).

It is likely that some cases of facial palsy are not due to pressure of the forceps blade on the nerve but rather to ischaemic damage within the facial nucleus; Gaisford (1953) has pointed out that this palsy may occur after normal delivery. Central ischaemic damage may also be the cause of congenital facial diplegia for which no adequate explanation has ever been offered other than agenesis of the brain stem, presumably genetic in origin (Murphy and German, 1947). It is pertinent that Hellström (1949) found other cranial nerves to be involved frequently in this condition. In $64 \%$ the abducent and in $23 \%$ the hypoglossal were affected. In $10 \%$ there was some mental defect.

At the upper end of the brain stem the nuclei of the third, fourth and sixth nerves will be damaged by vertebral arterial ischaemia, providing an explanation for the observation of Guibor (1950) that over half the patients with cerebral palsy have imbalance of extra-ocular muscles. This might be a fully 
developed squint or a latent lesion, as in the cases reported by Breakey (1955) who found esotropia in $25 \%$ and exotropia in $7 \%$ of 100 spastics. Guibor (1953) reported a case of fixed bilateral nasal squint associated with hypotonicity of the limbs suggesting a concomitant cerebellar lesion. A clear history of trauma resulting from rotation of the neck during forceps delivery was found by Lebensohn (1955) in an infant with Perinaud's syndrome. The fixed pupils and loss of upward gaze probably resulted from ischaemia of the quadrigeminal plate region.

The vertebral arteries supply not only the auditory and vestibular nuclei of the brain stem but via the internal auditory arteries they supply the inner ear; some degree of deafness may be expected in those who survive birth injury to the cervical portion of these vessels. Auditory function is certainly disturbed in many spastics.

There is considerable evidence to suggest that stenosis or occlusion of the vertebral arteries at birth is an important factor in the production of cerebral palsy. Analogy with the lesions found in vertebral arterial occlusion in adults (Hutchinson and Yates, $1956,1957)$ indicates that bilateral symmetrical lesions of calcarine occipital cortex might be expected. Indeed Norman (1953) found that five out of 45 cases of cerebral palsy had been characterized clinically by cortical blindness and showed bilateral calcarine occipital gyral damage. Jéquier, Dufour and Rabinowicz (1957) reported the findings in a 10-year-old blind spastic idiot. In this case also, bilateral calcarine cortical ulegyria and sclerosis of the pulvinar were observed but were attributed to a lesion of the vein of Galen. The symmetry of some of the lesions in cerebral palsy has been interpreted in a variety of ways. Penrose (1949) felt that it must indicate a genetically determined hereditary cause. Benda (1952) stated that 'the symmetry of lesions depended on the one common structure which is equally shared by both hemispheres-the venous drainage into the great vein of Galen'. Many of the findings would be better explained by a consideration of the unique arterial union of the vertebral arteries which, as mentioned earlier, results in symmetrical blood supply of the brain stem, cerebellum and via the posterior cerebral arteries, the occipito-temporal cerebral cortex.

There is a sub-group of cerebral palsy, known as the atactic-cerebellar type, who commonly show normal mental function and in whom the pathological changes are confined to cerebellum and brain stem. It is difficult to find any alternative explanation for such a condition other than vertebral arterial occlusion produced by birth trauma.

Epilepsy is common in cerebral palsy. Faber
(1947) reported its occurrence in $48 \%$ of 99 cases. In general a large proportion of epilepsy has been attributed to lesions in the temporal lobe (Bailey and Gibbs, 1951). Falconer (1958) reported scarring of the ammon's horn, uncus and amygdala in 21 of 50 cases operated on for temporal lobe epilepsy. The age of onset of attacks in this group was $4 \frac{1}{2}$ years and it has been suggested that natal injury is responsible for the damage in epilepsy of this type (Earle, Baldwin and Penfield, 1953). These authors demonstrated that compression of the brain at birth produced herniation of parts of the temporal lobe through the tentorial opening with damage and scarring of the hippocampal region. Lindenberg (1955) emphasized that arterial ischaemia resulting from compression of branches of the posterior cerebral artery might be important. Much of the pathological change found in these cases of epilepsy lies within territory ultimately dependent on the vertebral arteries. It is possible therefore that bruising of these vessels in their cervical course may be an additional factor in producing such scars.

\section{Summary}

The necks of 60 infants dying in the perinatal period were examined histologically for evidence of birth trauma. The cases were selected at random from 213 stillborn and neonatal deaths occurring in 2,800 births. They included eight breech deliveries and three by caesarean section; 16 were stillborn.

In 27 cases evidence was found of distortional trauma to the cervical spine in the form of extraand sub-dural haemorrhage, haemorrhage into joint capsules and torn ligaments and dura.

A significant degree of traumatic damage to the spinal cord was found in only two cases, both breech deliveries.

In nine cases there was bruising and tearing of spinal nerve roots.

In 24 cases, following all methods of delivery, there were haemorrhages into the adventitial coat of one or both vertebral arteries. These haematomas were often of considerable size, narrowing the lumen of the vessel. They were thought to arise from tearing of the arterial twigs, supplying nerve roots and spinal cord, at their origins from the vertebral arteries.

In one infant, who died 12 days after birth, the left vertebral artery was occluded by thrombus.

The significance of stenosis of the cervical part of the vertebral arteries is discussed in relation to ischaemic damage to the brain stem and as a probable cause of cerebral lesions in many cases of cerebral palsy. 
I would like to thank Dr. F. A. Langley and Dr. T. Wade-Evans for allowing me to carry out this special examination of cases on which they were performing a general autopsy.

\section{REFERENCES}

Baar, H. S. (1946). Brit. med. Bull., 4, 178.

Bailey, P. and Gibbs, F. A. (1951). J. Amer. med. Ass., 145, 365.

Benda, C. E. (1952). Developmental Disorders of Mentation and Cerebral Palsies. Grune and Stratton, New York.

Breakey, A. S. (1955). A.M.A.Arch Ophthal, 53, 852.

Chandler, F. A. (1948). J.M. Bone Jt Surg., 30A, 566.

Crothers, B. and Putnam, Marian, C. (1927). In Birth Injuries of the Central Nervous System, by Ford, F. R Crothers, B and Putnam, M. C. Medicine Monographs XI. Baillière, Tindall and Cox, London.

Earle, K. M., Baldwin, M. and Penfield, W. (1953). A.M.A. Arch. Neurol. Psychiat., 69, 27 .

Faber, H. K. (1947). Amer. J. Dis. Child., 74, 1

Falconer, M. A. (1958). Proc. roy. Soc. Med., 51, 613

Ford, F. R. (1925). Arch. Neurol. Psychiat. (Chicago), 14, 742.

Gaisford, W. (1953). In Paediatrics for the Practitioner, Vol. 1, p. 80.
Ed. by W. Gaisford and R. Lightwood. Butterworth, London: Guibor, G. (1950). Med. Clin. N. Amer., 34, 283.

- (1953). Amer. J. Ophthal., 36, 1719

Hausbrandt, F. and Meier, A. (1935). Frankfurt. Z. Path., 49, 21.

Hellström, B. (1949). Acta paediat. (Stockh.), 37, 464.

Himwich, H. E. and Fazekas, J. F. (1941). Amer. J. Physiol., 132, 454.

Hirvensalo, M. (1949). Acta paediat. (Stockh.), 37, Suppl. 1.

Hutchinson, E. C. and Yates, P. O. (1956). Brain, 79, 319.

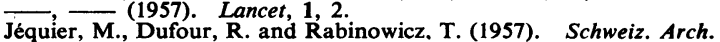
Neurol. Psychiat., 79, 170.

Lebensohn, J. E. (1955). Amer. J. Ophthal., 40, 738.

Lindenberg, R. (1955). J. Neuropath. exp. Neurol., 14, 223.

Little, W. J. (1843). Lancet, 1843-44, 1, 318.

- (1862). Trans. obstet. Soc. Lond., 1861, 3, 293.

Murphy, J. P. and German, W. J. (1947). Arch. Neurol. Psychiat (Chicago), 57, 358.

Norman, R. M. (1953). Proc. roy. Soc. Med., 46, 627

Penrose, L. S. (1949). J. ment. Sci., 95, 373.

Pierson, R. N. (1923). Surg. Gynec. Obstet., 37, 802.

Racker, E. (1942). Fed. Proc., 1, 69.

Radford, T. (1834). Lond. Med. Surg. J., 5, 144.

Roemer, F. J. (1954). Amer. J. Obstet. Gynec., 68, 1146 